\title{
Repeated Social Defeat Stress Induces Neuroinflammation and Impairs Hippocampal Neurogenesis That Differentially Regulate M ood and Cognition
}

A nzela Niraula, G raduate Student A ssociate

N euroscience Graduate Program, The Ohio State U niversity, Columbus, OH 43210

\section{Abstract}

Repeated social defeat (RSD) is a murine stressor that models several key physiological, immunological, and behavioral al terations observed in humans exposed to psychosocial stress. RSD induces prolonged anxiety-like behavior associated with myeloid cell trafficking into the brain, including the hippocampus. Because the hippocampus (HPC) is a key area involved in neuroplasticity, behavior, and cognition, the goal of this study was to investigate if the stressinduced monocyte trafficking affected hippocampal neurogenesis and cognitive function. Here, we show that RSD increased inflammatory mediators (IL-1 $\beta$, TNF $\alpha$ and IL -6), enhanced microglia activation and monocyte trafficking (CD 45hi) specifically in the caudal hippocampus. RSD also impaired spatial memory recall in the B arnes maze independent of anxiety-like behavior. RSD did not affect the number of proliferating neural progenitor cells and developing neurons when examined 14 hours post-RSD. Nonetheless there was a significant reduction in the number of young neurons and mature neurons in the HPC 10 days and 28 days post-RSD, respectively. Consistent with region-specific neuroinflammation, reduction in the number of mature neurons was greater in the caudal hippocampus of the RSD mice compared to controls. The RSD-induced spatial deficits, which are rostral hippocampus-mediated, were resolved by 28 days. Social avoidance which is caudal hippocampus-mediated still persisted 28 days after stress. Thus, stress-induced neuroinflammation is associated with reduced neuroplasticity, and the stress-induced affective and cognitive deficits are differentially associated with hippocampal neurogenesis. 


\section{Introduction}

Psychological stressors promote inflammation and immune dysfunction [1] that are implicated in mental health disorders [2], and accelerated cognitive decline [3, 4]. We propose that stress-associated release and re-distribution of myeloid cells is an important link between immune dysfunction and development of mood disorders [5]. Under stressful stimuli, peripheral myeloid cells can produce pro-inflammatory cytokines [6] and readily traffic to the brain [7]. Indeed, we have previously reported trafficking of green fluorescent protein (GFP)-positive peripheral monocytes in bone marrow-chimera mice exposed to repeated social defeat (RSD). For example, there was a stress exposure dependent increase in monocytes in circulation and macrophage recruitment to the brain (perivascular and parenchymal) that corresponded with the development of anxiety. These cells exhibited a "primed" phenotype, marked by glucocorticoid insensitivity and heightened ability for pro-inflammatory cytokine production. Trafficking of these cells was instrumental in the induction of prolonged anxiety-like behavior [8-12].

In addition, mice lacking important chemokine receptors for monocyte trafficking, CCR2 and $C X_{3} C R 1$, had limited macrophage recruitment to the brain and did not develop anxiety-like behavior after RSD [7]. Stress-induced myeloid cell trafficking and inflammation was localized within the limbic system including the hippocampus. This is relevant because the hippocampus is a key area involved in neuroplasticity, behavior, and cognition [13]. Stressful experiences may lead to reduced hippocampal neurogenesis and induce hippocampal atrophy, often prevalent in depressed patients (Sheline et al., 2003). Indeed, increased neuroinflammatory signaling augments neurobiological processes implicated in many behavioral disorders including neurogenesis [14] and metabolism of neurotransmitters [15, 16]. Furthermore, inflammatory 
signaling induces oxidative stress, excitotoxicity, impaired neural plasticity and neuronal functioning, all of which are implicated in affective impairments [15].

In addition to monocyte trafficking into the brain, microglial activation is another key component of stress-induced neuroinflammation. In fact, activated microglia secrete proinflammatory cytokines and chemokines which most likely contribute to recruitment of peripheral monocytes into the brain [17]. Inflammatory cytokines in the CNS and periphery also induce microglia activation [18-20] and impaired spatial learning and memory [21]. M oreover, microglia activation and cytokine production regulate neuronal plasticity [22] and neurogenesis [23-25] in vivo and ex vivo.

Thus, microglial activation and peripherally-derived monocytes together orchestrate neuroinflammation following RSD. Given the implicated association between inflammation and neurogenesis [26], this paper investigates the cognitive and affective effects of RSD-induced neuroinflammation, and its role in hippocampal neurogenesis.

\section{Methods}

$\underline{\text { Animals }}$

M ale C57B L/6 (6-8 weeks old) and male CD-1 retired breeder mice were purchased from Charles River B reeding Laboratories (Wilmington, M A) and allowed to acclimate to their surroundings for 7-10 days before experiments. Mice were housed in $11.5^{\prime \prime} \times 7.5^{\prime \prime} \times 6 "$ polypropylene cages. Rooms were maintained at $21^{\circ} \mathrm{C}$ under a $12 \mathrm{~h}$ light: $12 \mathrm{~h}$ dark cycle from 0600-1800 hrs with ad libitum access to water and rodent chow. All experiments took place between 0800 and 1100 hrs, unless otherwise noted. All procedures were in accordance with the 
NIH Guidelines for the Care and U se of L aboratory A nimals and were approved by the Ohio State U niversity Institutional Laboratory A nimal Care and U se Committee. Repeated Social D efeat (RSD)

RSD was performed as previously described [27]. In brief, an aggressive male intruder CD-1 mouse was introduced into cages of established male cohorts (3 per cage) of C57BL/6 mice for 2 hours between 17:00 and 19:00 for six consecutive nights. During each cycle, submissive behavior including upright posture, fleeing, and crouching were observed to ensure that the resident mice were exhibiting subordinate behavior. If the intruder did not initiate an attack within 5-10 minutes or was attacked by any of the resident mice, then a new intruder was introduced. At the end of the $2 \mathrm{~h}$ period, the intruder was removed and the residents were left undisturbed until the following day when the paradigm was repeated. Different intruders were used on consecutive nights. The heal th status of the mice was carefully examined throughout the paradigm. M ice that were injured or moribund were removed from the study. Consistent with previous studies using RSD [8], less than $5 \%$ of mice met the early removal criteria. Control mice (CON) were left undisturbed in their home cages until sacrificed.

\section{Morris Water Maze}

Spatial memory [28] and working memory [29] were assayed in the M orris water maze as Previously described. The apparatus consisted of a white $250 \mathrm{~cm}$ diameter tub filled with a mixture of water and white paint (at room temperature). A clear plastic platform (diameter 10 $\mathrm{cm}$ ) was conceal ed $1 \mathrm{~cm}$ beneath the surface of the water, and was placed in a position $20 \mathrm{~cm}$ away from the edge of the tub. The area surrounding the apparatus consisted of two distinct extra-maze geometric cues as well as additional spatial cues inherent to the room. The 
experimenter and recording equipment were concealed behind a curtain during testing. B ehavior was recorded and analyzed by digital recording system (Noldus)

The spatial memory assay involved a 5 day acquisition phase and a probe trial on the 6th day. The acquisition phase consisted of four trials per day for 5 days, with the platform in the same position throughout the task. During the probe trial on the 6th day, the platform was removed and percent time in each quadrant was recorded. M aximum trial duration was 60 seconds. If the platform was not reached within 60 seconds, then the mouse was placed on the platform and a latency of 60 seconds was recorded. Mice were placed at one of four positions in a quasi-random order. Latency and total distance to reach the platform, and percent time in the outer annulus of the tub were recorded. During the probe trial on the sixth day, the platform was removed and total distance traveled and percent time in each quadrant was recorded for 60 seconds.

W orking memory was assayed in an adaptation of the M orris water maze [29]. All conditions were the same as described above, unless otherwise noted. There were three massed trials per day with an inter-trial interval of $30 \mathrm{~s}$ and maximum trial duration of $120 \mathrm{~s}$. Each trial lasted until the platform was reached or until the maximum trial duration elapsed. If the platform was not reached within $120 \mathrm{~s}$, then the mouse was placed on the platform and a score of $120 \mathrm{~s}$ was recorded. In a pseudorandom and experimentally balanced manner, mice were placed in one of four quadrants approximately $3 \mathrm{~cm}$ from the edge of the tub. The location of the platform was changed every day, but remained in the same place during trials on the same day. A verage velocity, latency to platform, distance to platform, and duration spent in the outer annulus were recorded. 


\section{Barnes maze}

To evaluate hippocampal dependent spatial learning, the Barnes maze paradigm as described (Sunyer et al 2007) was employed with a few modifications. Acquisition phase consisted of four trials per day for four consecutive days. M ice were placed in the center of the maze under a semi-opaque container for 15 seconds (s). The trial starts recording when the container is lifted. Each trial lasted $180 \mathrm{~s}$ or until the mouse entered the escape hole. If the mouse did not reach the escape hole in the allotted time then it was guided to the hole. Speed, distance traveled, time to find the escape hole, and the number of errors were determined. Following six cycles of RSD, mice were tested for immediate recall $24 \mathrm{hrs}$ and $48 \mathrm{hrs}$ after the final day of stress. Long-term spatial recall was tested 28 and 29 days after the final day of stress.

$\underline{\text { Social Avoidance }}$

Social avoidance was determined as previously described [30]. In the empty trial, an experimental mouse was placed into the arena with an empty wire mesh cage and activity was recorded for 2.5 minutes. In the social trial, an unfamiliar CD-1 mouse was placed in the wire mesh cage and the experimental mouse was placed in the arena and activity was recorded for 2.5 minutes. A ctivity was video-recorded and analyzed using N oldus EthoV ision Software (L eesburg, V irginia).

Bromodeoxyuridine (BrdU) Injection

M ice were injected with $50 \mathrm{mg} / \mathrm{kg} \mathrm{BrdU}$ at 16:00 (1 hr before social defeat) on the last three nights of RSD (i.e., cycles 4, 5, \& 6). B rains were collected for B rdU immunohistochemistry at 14 hours, 10 days, or 28 days after the last cycle of RSD. 
Immunohistochemistry.

B rains were collected from mice after transcardiac perfusion with sterile PBS followed by $4 \%$ formaldehyde. Brains were post-fixed in $4 \%$ formaldehyde for 24 hrs followed by $30 \%$ sucrose for $48 \mathrm{hrs}$. Fixed brains were then frozen in isopentane $\left(-78^{\circ} \mathrm{C}\right)$ and sectioned at $25 \mu \mathrm{m}$ using a M icrom HM 550 cryostat, and used for immunofluorescent analyses. B rain regions were identified by reference markers in accordance with the stereotaxic mouse brain atlas [31]. $\underline{\text { Immunolabeling of CD } 45 \text { and Iba1-positive cells }}$

Immunofluorescent studies for CD 45 and Iba-1 were performed with rat anti-CD 45 (A bcam, 1:500) and rabbit anti-Ibal (W ako, 1:1000) respectively. Free-floating sections were blocked with 5\% normal donkey serum for 1 hour at room temperature. Primary and secondary antibody incubation lasted 18 hours each at $4^{\circ}$. The secondary antibodies were donkey anti-rat 488 (A lexafluor, 1:500) and donkey anti-rabbit 594 (A lexafluor, 1:500). M ounted sections were dried and then cover-slipped with Fluoromount (B eckman Coulter, Inc., Fullerton, CA) and stored at $-20^{\circ} \mathrm{C}$. Sections were imaged using a confocal laser scanning microscope. The values for Ibal proportional area and number of CD 45-positive cells represent bilateral hippocampi in each label ed section.

\section{$\underline{B r d U}$ and doublecortin labeling}

For B rdU labeling, sections were washed, denatured in $2 \mathrm{~N} \mathrm{HCL}$ at $37^{\circ} \mathrm{C}$ for $30 \mathrm{~min}$, and blocked with 3\% normal goat serum. Sections were labeled using rat anti-B rdU (A bD Serotec, $\mathrm{mA} \mathrm{b} 1: 1000$ ) at $4^{\circ} \mathrm{C}$ for $24 \mathrm{hr}$. The secondary antibody was goat anti-rat 488 (A lexafluor, 1:500), and secondary incubation was performed at $4^{\circ}$ overnight. Doublecortin labeling was performed using 5\% normal donkey serum for blocking, anti-doublecortin (A bcam, 1:500) for primary antibody, donkey anti-goat 594 (A bcam, 1:500) for secondary antibody incubation. The 
numerical values for B rdU-positive cells and doublecortin-positive cells were calculated for the entire hippocampus.

\section{BrdU and doublecortin/NeuN/GFAP double-labeling}

For phenotypic analysis of $\mathrm{BrdU}^{+}$cells at 10 and 28 days post injection, double labeling was performed with rat anti-B rdU (A bD Serotec, mA b 1:1000), goat anti-doublecortin (A bcam, 1:500) and rabbit anti-N euN (A bcam, 1:1000) or rabbit anti-GFA P (Dako, Rabbit1:1000). Immunofluorescent-labeled sections for the B rdU and doublecortin labeling were visualized using confocal laser scanning microscope. Sections collected at 10 days and 28 days postinjection were visualized using wide field fluorescent microscope. Total number of cells with positive labeling in the hippocampus was estimated by multiplying by the number of cells by two, to represent the bilateral hippocampi in each brain slice.

\section{$\underline{\text { RNA isolation and real time PCR }}$}

B rain tissue samples were collected from the hippocampus and cortex both immediately after and $14 \mathrm{~h}$ after the final cycle of RSD. RNA was isolated from homogenized brain regions using Tri-reagent//sopropanol precipitation, RNA was reverse transcribed to CDNA using an RTRETROscript kit (A mbion, A ustin, TX), and RNA concentration was determined by spectrophotometry. Quantitative PCR was performed using the A pplied Biosystems (Foster, CA) A ssay-on-D emand Gene Expression protocol as previously described. In brief, experimental CDNA was amplified by real-time PCR where a target CDNA (e.g., IL-6, BDNF, VEGF) and a reference cDNA (glyceral dehyde-3-phosphate dehydrogenase; GAPDH) were amplified simultaneously using an oligonucleotide probe with a $5^{\prime}$ fluorescent reporter dye (6-FA M ) and a $3^{\prime}$ quencher dye (NFQ or TAM RA ). Fluorescence was determined on an A BI PR ISM 7300sequence detection system (A pplied B iosystems, Foster, CA ). Data were analyzed using the 
comparative threshold cycle $(\mathrm{Ct})$ method and results are expressed as fold difference from GAPDH normalized to controls.

$\underline{\text { Statistical analysis }}$

To ensure a normal distribution, data were subjected to Shapiro-W ilk test using Statistical A nalysis Systems (SA S) statistical software (Cary, NC). O bservations greater than 3 interquartile ranges from the first and third quartile were considered outliers and were excluded in the subsequent analysis. To determine significant main effects and interactions between main factors, data were analyzed using one- or two-way A N OV A using the G eneral Linear M odel procedures of SAS. When there was a main effect of experimental treatment, differences between group-means were evaluated by an F-protected t-test using the Least-Significant Difference procedure of SAS. A Il data are expressed as treatment means \pm standard error of the mean (SEM).

\section{Results}

Increased inflammation and presence of CD 45-positive myeloid cells in the hippocampus after RSD.

We have previously reported that RSD activated microglia and enhanced the trafficking of bone marrow derived myeloid cells to the brain [7]. M oreover, these events contributed to stress-induced neuroinflammation, IL-1 signaling, and the development of prolonged anxietylike behavior. Stress induced myeloid cell trafficking and inflammation was localized within the limbic system including the hippocampus. This is relevant because the hippocampus is a key area involved in neuroplasticity, behavior, and cognition. Therefore, the objective of this study was to determine the effect of RSD on hippocampal dependent memory and neuronal plasticity. 
In the first experiment, mice were exposed to six cycles of repeated social defeat (RSD) and immediately after the last cycle of social defeat, the hippocampus was collected and mR NA expression of several inflammatory mediators and growth factors were determined. Fig.1A shows that mRNA levels of IL-12 $(p<0.0001)$, IL $-6(p<0.05)$, TNF $\alpha(p<0.05)$ were all increased in the hippocampus immediately after RSD. These data are consistent with our previous findings [27]. In addition, mRNA expression of nerve growth factor (NGF), $(p<0.05)$ and vascular endothelial growth factor (VEGF) $(p<0.01)$ were also increased in the hippocampus of RSD mice compared to controls. A rginase, brain-derived neurotrophic factor (BDNF), and insulin-like growth factor-1 (IGF1) were unaffected by RSD.

Next, microglial 1 ba-1 immunoreactivity and the presence of CD 45-positive monocytes were assessed in the dentate gyrus (DG) of the hippocampus. Fig1B shows representative images of Iba-1 labeling of microglia in the DG. As expected, microglial Iba-1 proportional area in the D G was increased by RSD compared to controls $(F(1,19)=11.66, p<0.05 ; \mathrm{Fig} .1 \mathrm{C})$. The microglia in the DG had a de-ramified morphology compared to controls, consistent with an increased inflammatory profile of microglia after RSD (refs). M oreover, increased iba-1 immunoreactivity was significantly higher in the caudal hippocampus of the RSD mice compared to the rostral hippocampus of RSD mice $(p<0.05)$. Fig.1D shows representative images of CD 45-positive labeling of monocytes/macrophages in the DG of control and RSD mice. There was a significant increase in the number of CD 45-positive peripheral myeloid cells in the $D G$ of the hippocampus with stress $(F(1,20)=4.76, p<0.05 ; F i g .1 E)$. The $D G$ was also subdivided into caudal and rostral sections and CD 45 cells were counted. The increase of CD 45+ monocytes was more pronounced in the caudal hippocampus of the RSD mice than the rostral hippocampus $(p<0.05)$. Overall, these data indicate that RSD increases inflammation, 
microglial Iba-1 immunoreactivity and number of CD 45-positive monocytes in the caudal hippocampus.

\section{RSD impaired working memory in the Morris water maze}

Inflammatory cytokines may act as negative regulators of hippocampal-dependent memory. For example, peripheral LPS injection impaired spatial working memory [29] and hippocampal IL-1 impaired spatial memory recall [32]. Therefore, inflammation in the HPC associated with RSD may reduce hippocampus-dependent memory functions. To test this idea, mice were exposed to six cycles of repeated social defeat (RSD) and working memory was assessed [29]. Here, a working memory adaptation of the M orris water maze (M W M ) was used. In this design, mice were trained to locate the hidden platform that changed location on each day of testing (Fig.2A). Fig.2B shows representative search patterns of CON and RSD mice on day 3 of testing in the M W M. RSD did not affect swim velocity during the five days of testing (Fig.2C). RSD, however, increased the latency of mice to reach the platform on test days 1,2 and $3(F(1,95)=4.38, p<0.05 ; F i g .2 D) . R S D$ al so tended to increase the distance traveled across test days $1-3(F(1,95)=3.39, p<0.1 ; F i g .2 E)$. By day 4 , however, latency and distance travelled were no longer affected by RSD. It is important to note that an anxiety-like search strategy (i.e, thigmotaxis) was observed in RSD mice on test days 1-3 (Fig.2F). M ice subjected to RSD spent more time in the outer annulus of the maze compared to controls $(F(1,95)=10.63, p=0.0001$; Fig. 2E). This thigmotactic search strategy was al so apparent in the representative search paths represented in Fig.2B. Taken together, RSD impaired working memory in the M orris water maze in a time dependent manner, but these differences also coincided with an anxiety-like search strategy. 


\section{RSD impaired spatial recall but not acquisition in the M orris water maze}

To further assess cognitive functioning, another version of the M W M was used to test spatial memory recall. In this experiment, mice were trained in the M WM to acquire the location of the hidden platform (Fig.3A) on the first five days following the six cycles of RSD (A cquisition phase). Then, memory was probed on the sixth day with removal of the hidden platform, and latency to the platform (Fig.3B) and distance travelled (Fig.3C) to the platform were determined.

During the acquisition phase 5 days after RSD, neither latency to the platform (Fig.3B) nor distance travelled were affected by RSD (Fig.3B). Thus, both groups learned the location of the platform during acquisition. During the probe trial, however, control mice outperformed the RSD mice in memory recall. Representative paths from the probe trial are shown for CON and RSD mice in Fig.3D. M ice exposed to RSD spent significantly less time in the target quadrant compared to controls ( $p<0.05 ; \mathrm{Fig.3F}$ ). The reduced time spent in the target quadrant by the RSD mice in the probe trail was not associated with increased total distance traveled (Fig.3E). Collectively, RSD impaired spatial memory recall in the M orris water maze probe trial.

\section{I mpaired spatial memory recall in the B arnes maze after RSD was not associated with anxiety, impaired encoding, or deficient memory consolidation.}

Because RSD-induced anxiety-like behavior persists for at least 8 days [33], it is possible that decreased time spent in the target quadrant of the M orris water maze is attributable to increased anxiety-like behavior or reduced encoding efficiency. In order to circumvent the possible anxiety- or encoding-related deficits, spatial memory was assessed in the B arnes maze. In this design, mice were allowed to acquire the location of the escape hole in the Barnes maze 
for four days prior to the beginning of RSD, exposed to RSD for 6 days, and memory recall was determined on the $7^{\text {th }}$ and $8^{\text {th }}$ day or the $28^{\text {th }}$ and $29^{\text {th }}$ day (Fig.4A).

As expected, there were no differences in the number of errors, latency, or distance traveled (not shown), on any acquisition day mice tested prior to RSD (pre-CON and pre-RSD). M ice were tested on the Barnes maze for two consecutive days after the $6^{\text {th }}$ cycle of social defeat (days 7 and 8). RSD significantly increased latency to find the escape ( $p<0.05$; Fig. 4B). There was also an increased number of errors ( $p<0.05 ; \mathrm{Fig} .4 \mathrm{C}$ ) and increased distance traveled (data not shown) to reach the platform during the recall phase of the test on day 7 and day 8 after RSD.

Next, a similar paradigm was used to determine long-term memory recall in the Barnes maze 28 days after RSD. M ice were allowed to acquire the location of the escape platform for four days prior to RSD. No differences were observed in the number of errors, latency, or distance traveled between experimental groups on any acquisition day (Fig.4D\&E). Twentyeight days following RSD, CON and RSD mice were tested on the Barnes maze for two days (days 28 and 29). RSD had no effect on errors, latency or distance traveled on any of the two test days. It is important to note that all mice retained a strong spatial memory of the location of the escape hole even 28 days after the memory acquisition phase of testing. Collectively, these combined data imply that RSD caused transient deficits in spatial memory recall in the Barnes maze that were not associated with anxiety-like search strategies, impaired encoding, or deficient consolidation.

\section{No change in the number of proliferating progenitor cells or young neurons in the dentate gyrus 0.5 day after RSD.}


Cognitive impairments following stress have been previously associated with inflammation and impaired neurogenesis [34]. Therefore, given that RSD increased hippocampal IL $-1^{2}$ expression in the current study (Table 1 ), the effects of RSD on neurogenesis were determined. To mark proliferating neural progenitor cells (NPCs) in the dentate gyrus of the hippocampus, B rdU was injected on the last three days of RSD, and B rdU + cells in the dentate gyrus were determined 0.5 day after RSD. Fig.5A shows representative images of B rdU labeling in the dentate gyrus. The total number of B rdU + cells in the dentate gyrus did not differ significantly between RSD and control mice $0.5 \mathrm{~d}$ after stress (Fig.5B).

In a related study, the number of cells expressing doublecortin (DCX), an early neuronal marker, in the dentate gyrus was also determined. Fig.5C shows representative images of DCX labeling in the DG of control and RSD mice. At $0.5 \mathrm{~d}$ following RSD, there were no differences in the number of DCX positive cells between the treatment groups (Fig.5D). Next, phenotypic analysis of the DCX-positive cells was used to differentiate mature and immature neurons [35]. Representative images of a DCX labeled mature and immature neuron are shown (Fig.5E). A gain, there was no significant difference in the number of immature or mature neurons between RSD and CON at $0.5 \mathrm{~d}$ after RSD (Fig.5F). These results indicate that RSD did not affect the generation of new neurons during this time period.

\section{RSD reduced the number of young proliferating neurons in the dentate gyrus.}

Our findings indicate no initial difference in the number of new BrdU positive cells 0.5 day after RSD. N onetheless, the fate of these cells may differ with time after RSD. Therefore, to assess the effects of RSD on the phenotypic fate of the hippocampal NPCs, mice were injected 
with $B r d U$ on the final three days of stress and the expression of $D C X$ and $B r d U$ was determined in the DG 10 days after RSD (Fig.6).

Fig.6A shows representative double-labeling of $B$ rdU and DCX in the dentate gyrus 10 days after RSD. The insets highlight a B rdU +/DCX + cell from controls (left) and BrdU +/DCX cell in the RSD mice (right). The proportion of B rdU + cells that co-localized with DCX was reduced in mice exposed to RSD compared to the controls ( $p<0.05$; Fig.6B). Similar to the 0.5 $d$ results, the total number of BrdU-positive cells was unaffected by RSD (data not shown). Taken together, the number of proliferating early neurons ( $\mathrm{BrU}+/ \mathrm{DCX}+$ cells) was reduced in the DG of hippocampus 10 days after RSD.

\section{RSD impaired neuronal differentiation of hippocampal NPCs}

Because there was a reduction in B $\mathrm{rdU}+$ cells that co-localized with the early neuronal marker DCX (Fig.6B) 10 days after RSD, it is possible that RSD may have reduced the neurogenic capacity of hippocampal NPCs. To test this idea, mice exposed to RSD were injected with B rdU during the last three cycles of stress and the brain was collected and processed 28 days later. This time point allows enough time for the B rdU -labeled NPCs to fully express mature phenotypic markers including N euN (mature neurons) and GFA P (astrocytes). B rdU + cells from sections collected 28 days after RSD were colabeled for N euN (Fig.7C) and GFA P (Fig.7E). The total number of BrdU + cells at this time point was not different between CON and RSD groups (Fig. 7A\&B). N onetheless, the number of BrdU + cells that colocalized with the mature neuronal marker $\mathrm{N}$ euN was reduced in mice exposed to $\operatorname{RSD}(F(1,18)=96.54, p<0.05$;

Fig. 7D\&E). M oreover, this reduction in N euN +/B D rU + 28d after RSD was more pronounced in the caudal hippocampus compared to the rostral hippocampus $(p<0.05)$. No differences were 
detected in the number of BrdU + cells that colocalized with GFAP (Fig.7E\&F). Overall, the findings indicate that there was a decreased number of proliferating progenitor cells that developed a neuronal phenotype in mice exposed to RSD.

\section{RSD-induced social avoidance was maintained at 28 days post-stress}

Our neurogenesis results indicate significantly greater reduction in the number of mature neurons in the caudal hippocampus of RSD mice compared to controls. Long-term memory at 28 days was not significantly affected in the RSD mice. Consistent with current understanding of the functional association between the caudal hippocampus and affective processing (Fanselow and Dong, 2010), previous work from our lab and others has shown increased social avoidance following RSD (D onahue, et al., 2014; W ohleb et al., 2013). Therefore, to examine the long-term role of RSD-induced reduced neurogenesis in affective functioning, mice were tested on the social avoidance task 28 days after RSD. The test consisted of two trials, where interaction was first determined using an Empty trial followed by a Social trial (W ohleb et al. 2013). During the Empty trial, both CON mice and RSD mice spent similar duration of time in the interaction and the corner zone. (Fig. $8 \mathrm{~B} \& \mathrm{C})$. During the Social trial, however, mice exposed to RSD spent less time in the interaction zone (stress $x$ trial interaction, $F(1,27)=6.17, p<0.05$;

Fig.8B) and more time in the corner zone (stress $x$ trial interaction, $F(1,27)=9.08, p<0.01$;

Fig. $8 \mathrm{C}$ ). These results indicate that RSD induces social avoidance in mice that persists at least 28 days following stress. 


\section{Discussion}

We have reported that repeated social defeat (RSD) is a murine stressor that induces prolonged anxiety-like behavior associated with myeloid cell trafficking into the brain (REFS). Here, we extend these previous findings to show that RSD drives robust microglia activation and monocyte trafficking (CD 45hi) specifically in the caudal hippocampus. A ssociated with this was impaired spatial memory recall after RSD that was independent of anxiety-like behavior. While the number of proliferating neural progenitor cells and developing neurons was initially unaffected by RSD, there was a significant reduction in the number of young neurons and mature neurons when examined 10 days and 28 days post-RSD. Consistent with region-specific neuroinflammation, reduction in the number of mature neurons was more pronounced in the caudal hippocampus of the RSD mice compared to the rostral hippocampus. The RSD-induced spatial deficits, which are rostral hippocampus-mediated, were resolved by 28 days. Social avoidance, which is caudal hippocampus-mediated still persisted 28 days after stress. Thus, RSD-induced neuroinflammation is associated with reduced neuroplasticity, and the stressinduced affective and cognitive deficits are differentially associated with hippocampal neurogenesis.

One of the novel findings of the current study is that we detected 2-3 fold more CD 45 high monocytes in the hippocampus of RSD mice compared to controls. M oreover this increase in CD45 monocytes was specific to the caudal hippocampus. Although we have previously shown RSD-induced trafficking of GFP-positive monocytes into the hippocampus bone marrowchimera mice (REF), the current study shows regional differences in RSD WT mice. BM chimera mice may overestimate the percentage of cells that enter the CNS parachyema with stress. N onetheless, even in WT mice we have reported consistent influx of monocytes to the 
brain by flow and IHC after RSD. Consistent with the regional specific of monocytes, there was also increased de-ramified morphology and higher Iba-1 proportional area of microglia in caudal hippocampus of RSD mice. [36, 37]. The increased presence of monocytes and microglia in the hippocampus was associated with elevated IL $1^{2}$, TNF and II-6 mRNA following RSD (W ohleb et al., 2013). NGF (nerve growth factor) and VEGF (vascular endothelial growth factor) were also increased in the hippocampus. One explanation for an increase in grow th factors with stress is that IL - 1 have been associated with enhanced endothelial secretion of VEGF (Y irmiya \& Goshen, 2011). In addition, increased NGF may represent a protective feedback in response to stress, which has been previously documented (A vitsur, Stark, \& Sheridan, 2001; B erry, Bindocci, \& Alleva, 2012). Results confirm regional specificity of microglia activation that is paralleled with increased monocyte trafficking and neuroinflammation.

A nother key finding of the current study is that RSD impaired cognitive performance on hippocampal-dependent tasks in the M orris W ater $M$ aze and B arnes $M$ aze. Since memory impairments on the M orris W ater M aze could be influenced by anxiety, it was important to distinguish stress-induced memory impairments from stress-induced anxiety. N onetheless, impairment in memory recall was clearly detected on the $B$ arnes $M$ aze test as shown by increased latency to locate the escape hole and greater number of errors. Interestingly, these deficits were transient, and no longer present 28 days after RSD. It is also important to highlight that the peripheral monocytes/macrophages that had trafficked into the brain are no longer detected 28 days after RSD [30].

A nother important finding of the current study is that RSD disrupts neuroplasticity. It is important to note the impaired plasticity was not evident until fate mapping. RSD did not reduce the expression of neural progenitors or young neurons in the dentate gyrus when tested 0.5 day 
following RSD, indicating that impaired spatial memory recall was not associated with reduced B rdU-positive cells or doublecortin-positive cells. Fate mapping of proliferating cells revealed that RSD significantly reduced the number of B rdU-positive doublecortin-expressing cells 10 days after RSD. RSD also significantly reduced the number of B rdU-positive cells that developed into $\mathrm{N}$ euN -positive mature neurons 28 days later. These results indicate that RSD does not affect the number of proliferating neural progenitor cells but does affect the total number of neurons that are incorporated into the dentate circuitry. Since our memory assessment 28 days after RSD did not reveal any memory deficits, the long term impact of the reduced neuronal fate is unclear.

Stress-induced inflammation is sufficient to have a long term impact on plasticity. For example, chronic isolation stress has been shown to reduce neurogenesis and impair several measures of learning and memory in an IL-1 receptor dependent manner [34]. For example, mice exposed to chronic isolation stress showed increased expression of hippocampal IL $1^{2}$ concomitant with memory impairments, which were reversed upon transplantation of neural progenitor cells overexpressing IL-1ra (IL-1 receptor antagonist) into the hippocampus [34]. The RSD-induced inflammation and subsequent impairments could extend well beyond neurons to other cell types, such as, glial cells [25]. In fact, previous in vitro reports in rats have shown IL$1^{2}$ to reduce hippocampal neurogenesis by diverting the fate of neural progenitor cells away from neuronal phenotype towards an astrocytic phenotype, thus exerting an anti-neurogenic and pro-gliogenic effect [25]. In the current study, the number of newly formed astrocytes, as marked by GFAP/B rdU double-labeling, in the dentate gyrus did not vary with RSD when examined at the 28 day time-point. This finding further suggests that RSD-induced inflammation does not alter proliferation of neural progenitor cells (as indicated by B rdU-labeling at $0.5 \mathrm{~d}$ post-RSD), 
but most likely attenuates their differentiation into neurons (as indicated by reduction in doublecortin-positive labeling at 10 days post-RSD) and their integration into the dentate circuitry (as indicated by reduction in N euN-positive labeling at 28 days post-RSD).

The attenuation in the number of neurons was evident in the caudal hippocampus of RSD-exposed mice compared to the controls. This region-specific differential effect of social defeat stress on neurogenesis has al so been demonstrated in previous studies [38]. M oreover, existing literature exploring the functional dichotomy of the hippocampus implicates the rostral and the caudal hippocampus in cognitive and affective processing respectively [39]. The caudal hippocampus, which anatomically projects to the prefrontal cortex, bed nucleus of the stria terminalis and subcortical structures associated with the hypothalamic-pituitary-adrenal (HPA) axis, regulates emotional behavior [40]. Previous work from our lab and others has shown increased social avoidance (a behavior associated with affect and mood) following social defeat in mice $[41,42]$. Here, we have shown that RSD-induced enhancement in social avoidance is maintained even 28 days after stress, and that this maintenance temporally corresponds with reduced number of neurons in the caudal hippocampus. Contrary to our finding, a previous study showed that mice more susceptible to social avoidance following social defeat stress had increased neurogenesis than the mice less susceptible to social avoidance [43]. In the study, mice were given a single B rdU injection 24 hours after exposure to stress, whereas in our study, mice received daily BrdU injections during the last three days of stress. Therefore, given this temporal variation in labeling of cells, a direct comparison between the two studies is unlikely. In our study, induction of social avoidance following RSD is likely regulated by neuroinflammation, while the maintenance of this social avoidance could be mediated by reduced neurogenesis in the caudal hippocampus. 
In summary, we have shown here that repeated social defeat induces an inflammatory profile in the hippocampus, marked by trafficking of peripheral monocytes and microglial activation. Concurrently, these effects are associated with memory deficits and depressive-like behavior. RSD-induced neuroinflammation specifically impairs neuronal differentiation of the progenitor cells in the dentate gyrus of the caudal hippocampus, and is associated with persistent depressive-like behavior. 


\section{Figure 1}

A.

\begin{tabular}{|l|c|c|l|c|c|}
\hline \multicolumn{5}{|c|}{ mRNA Expression in Hippocampus (Fold $\boldsymbol{\Delta}$ ) } \\
\hline Gene & CON & RSD & Gene & CON & RSD \\
\hline IL-1 $\beta$ & $1.1 \pm 0.18$ & $6.8 \pm 0.40^{*}$ & BDNF & $1.1 \pm 0.11$ & $1.0 \pm 0.07$ \\
TNF- $\alpha$ & $1.0 \pm 0.10$ & $3.3 \pm 0.71^{*}$ & VEGF & $1.0 \pm 0.08$ & $1.4 \pm 0.06^{*}$ \\
IL-6 & $1.0 \pm 0.09$ & $1.7 \pm 0.25^{*}$ & NGF & $1.0 \pm 0.11$ & $1.6 \pm 0.05^{*}$ \\
Arg 1 & $1.1 \pm 0.17$ & $1.1 \pm 0.11$ & IGF1 & $1.0 \pm 0.02$ & $0.9 \pm 0.02$ \\
\hline
\end{tabular}

B.

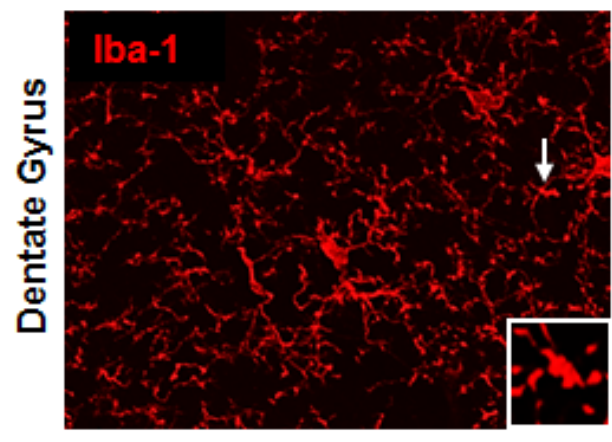

D.

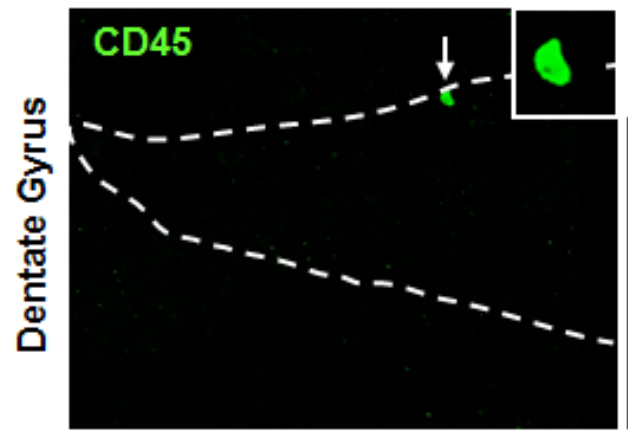

RSD

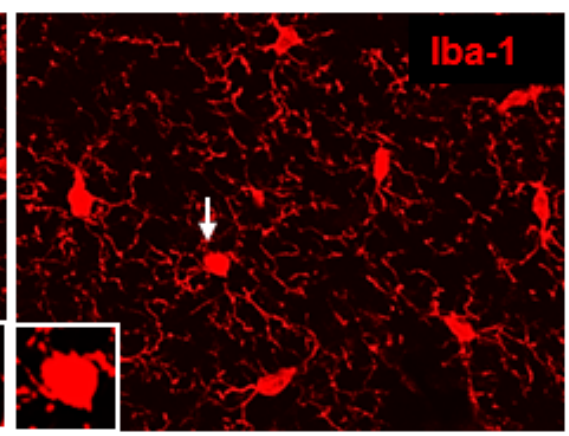

RSD

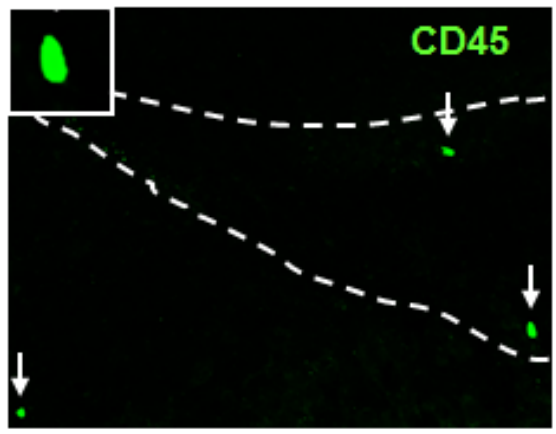

C.

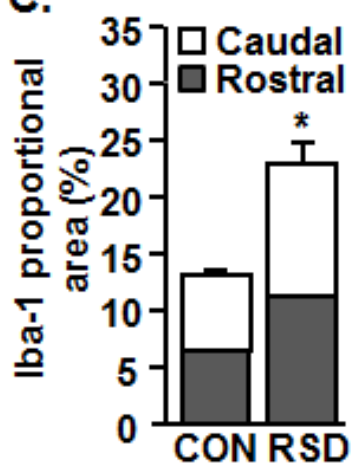

E.

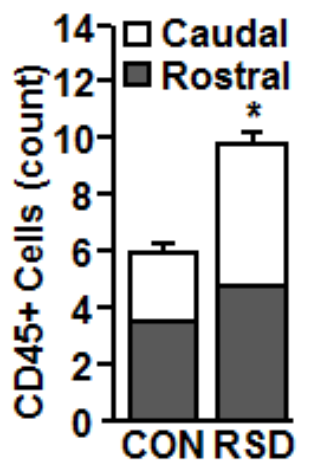

Increased Inflammation in the Hippocampus after RSD. M ice were subjected to 6 cycles of social defeat or left undisturbed as controls. A) M ice were sacrificed immediately after the sixth cycle of stress and mRNA expression of several inflammatory mediators (IL-1, TNF, IL-6, A rg) and growth factors (BDNF, VEGF, NG F, IGF-1) were determined in the hippocampus. In a related experiment, mice were subjected to 6 cycles of social defeat and brains were collected 14 h later. B) Representative images and C) quantification of microglial Iba-1 labeling are shown. D) Representative images and E) quantification of CD 45 labeling in the dentate gyrus are shown. Data represent mean \pm SEM. M eans with asterisk $(*)$ are significantly different from CON $(p<$ $.05)$ and means with (\#) tended to be different from CON $(p<.06-.10)$. 
Figure 2

A.
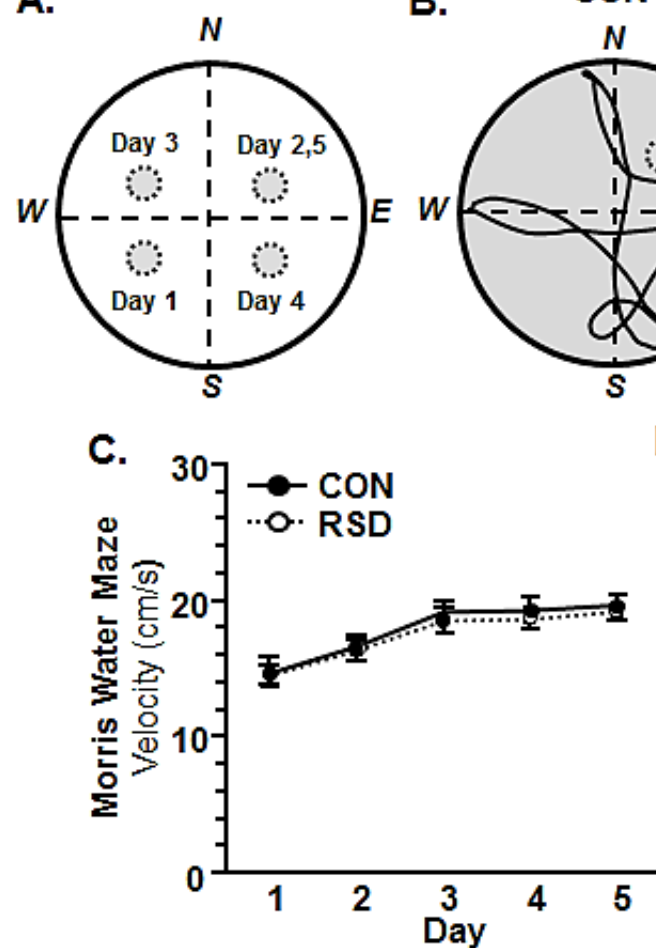

B. $\quad \operatorname{CON}$
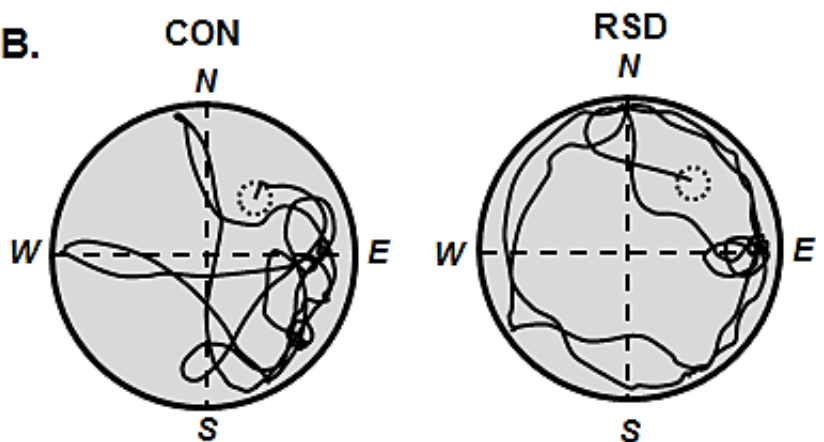

D.
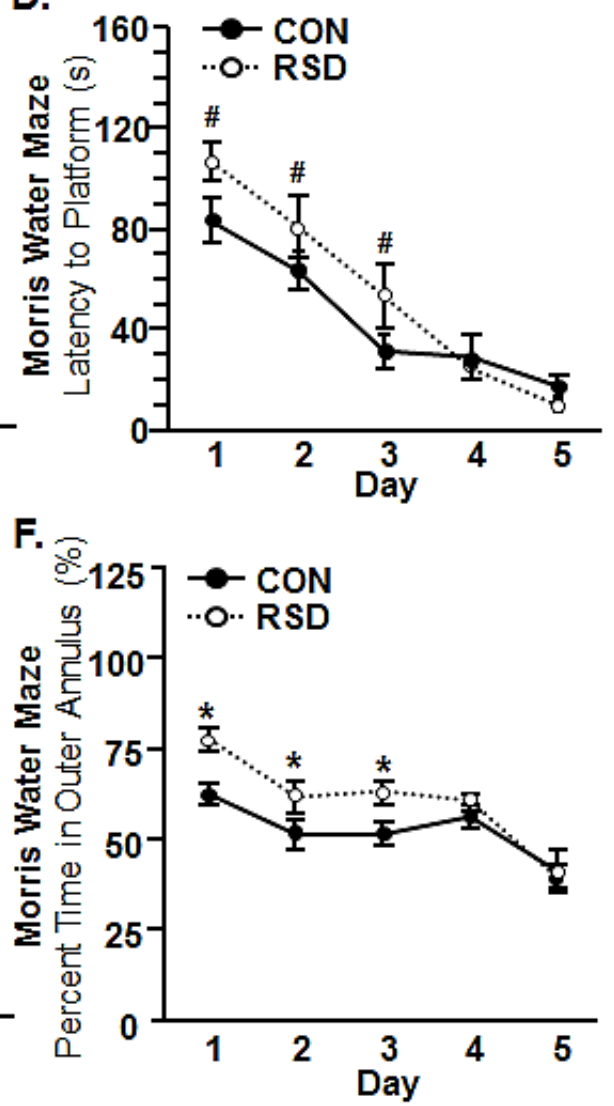

RSD impaired working memory in the Morris water maze. M ice were subjected to 6 cycles of social defeat or left undisturbed as controls. A) M ice were tested in the working memory variation of M orris water maze for 5 days after the completion of RSD. In this version, the platform is moved each testing day. Representative paths for B) CON and C) RSD mice in the water maze on day 3 are shown. C) V elocity D) latency to the platform, E) distance travelled, and $\mathrm{F}$ ) percent time in the outer annulus for all 5 testing days are shown. Lines represent the mean \pm SEM . M eans with asterisk $(*)$ are significantly different from CON $(p<.05)$ and means with (\#) tended to be different from CON $(p<.06-.10)$. 


\section{Figure 3}

A.

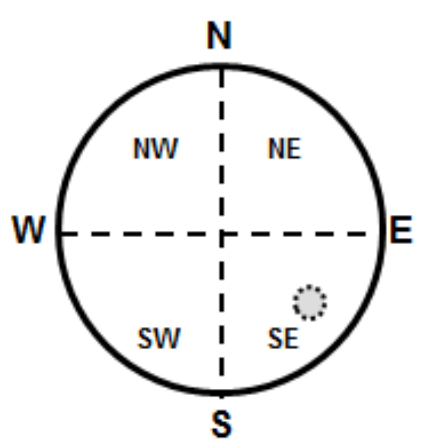

B.

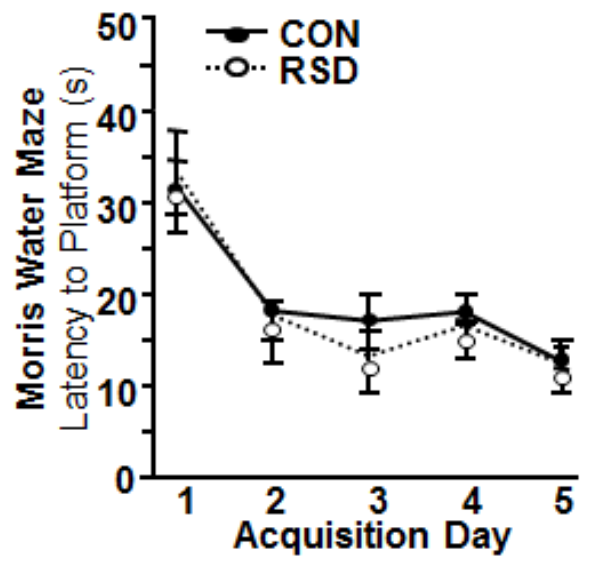

C.

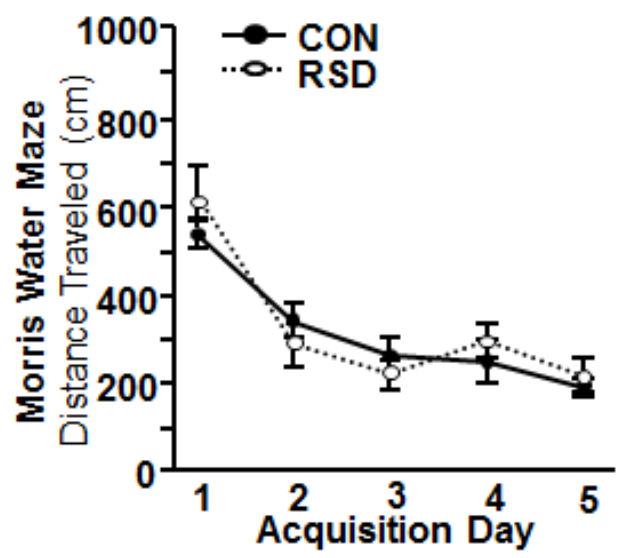

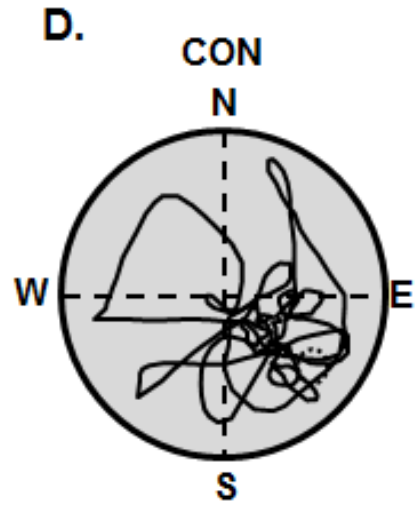

E.

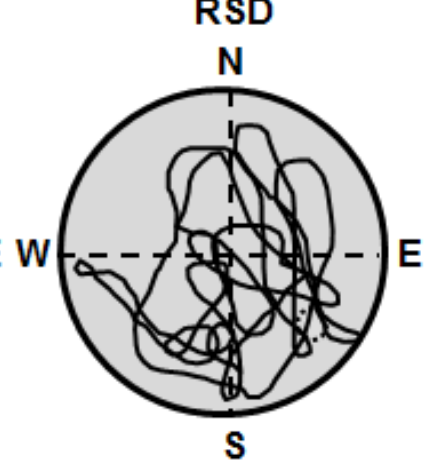

G.

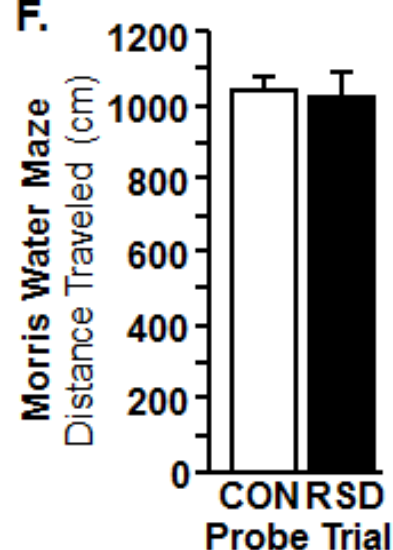

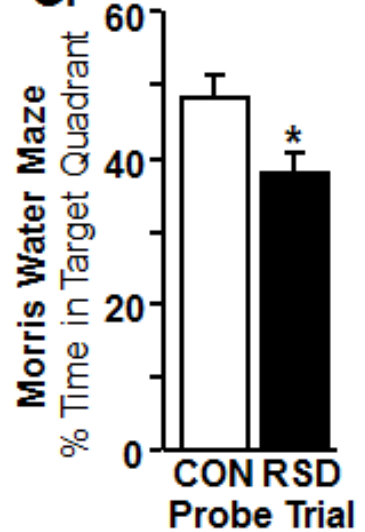

RSD reduced time spent in the target quadrant on the probe trial of the M orris water maze. $M$ ice were subjected to 6 cycles of social defeat or left undisturbed as controls. A) M ice were tested in the spatial memory variation of M orris water maze for 5 days after the completion of RSD. In this version, the platform remains in the same location each testing day. B) latency to the platform and $\mathrm{C}$ ) distance travelled for all 5 testing days are shown. Representative search paths for D) CON and E) RSD mice during probe trial. F) Distance traveled and G) duration spent in target quadrant during probe trial are shown. Lines and bars represent the mean $\pm S E M$. M eans with asterisk $(*)$ are significantly different from CON $(p<.05)$. 


\section{Figure 4}



B.

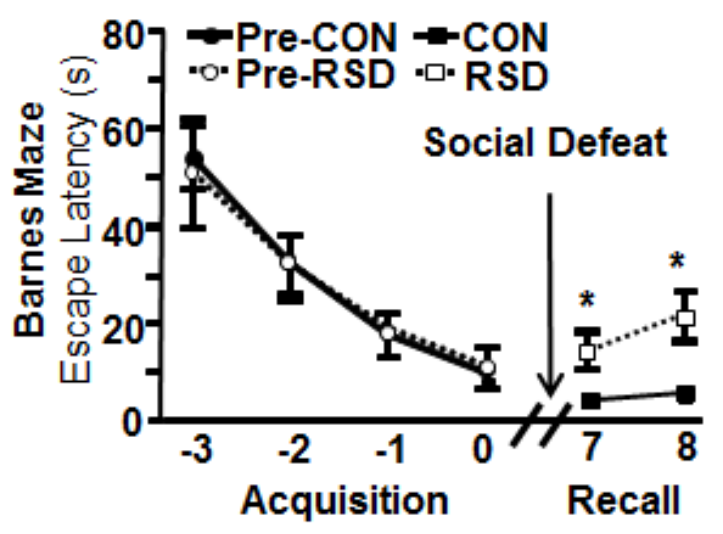

D.

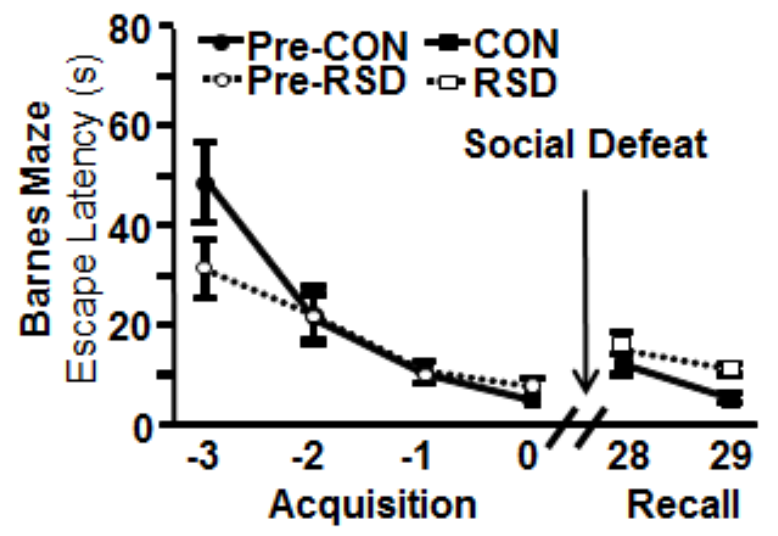

C.

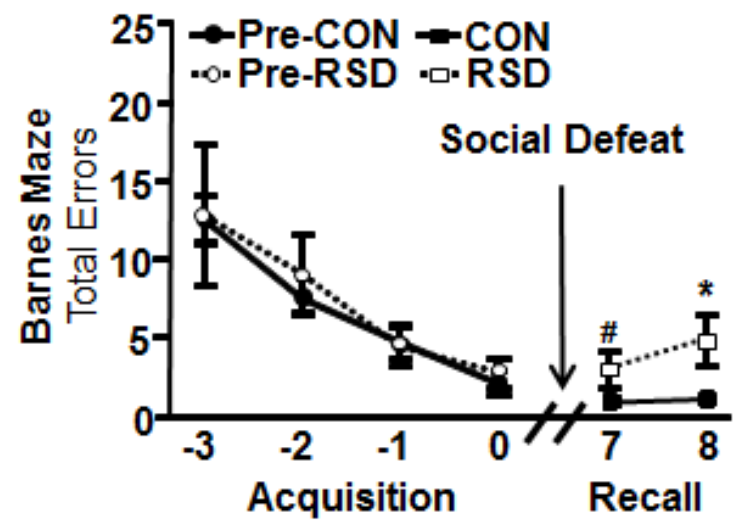

E.

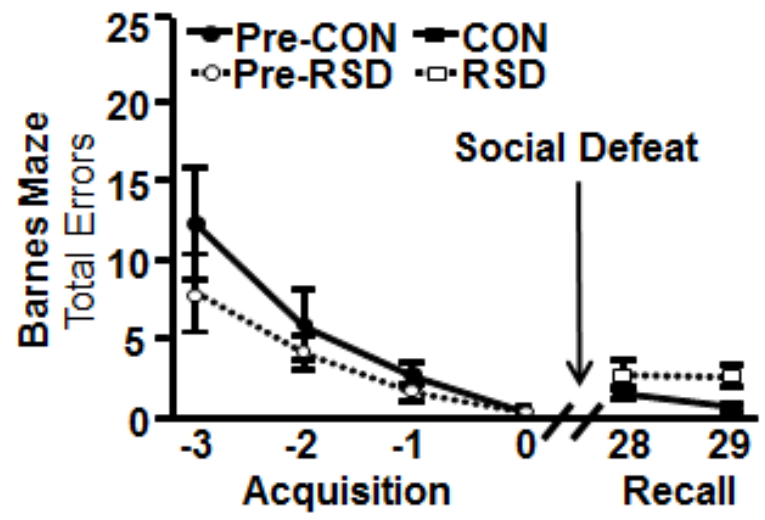

R SD impaired spatial learning on the Barnes maze. A) M ice were trained on the Barnes maze to acquire learning prior to RSD. M emory recall was determined 1, 2 or 28, 29 days after the last cycle of social defeat. B) Escape latency and C) total errors are shown before and 1 and 2 days after RSD exposure. D) Escape latency and E) total errors are shown before and 28 and 29 days after RSD exposure. Lines represent the mean \pm SEM . M eans with asterisk $\left(^{*}\right)$ are significantly different from CON $(p<0.05)$ and means with (\#) tended to be different from CON ( $p=0.06$ $0.10)$. 


\section{Figure 5}

A. $\quad \operatorname{CON}(0.5 \mathrm{~d})$

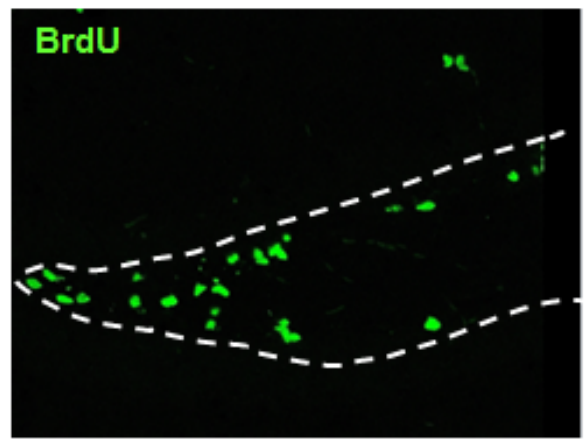

C.



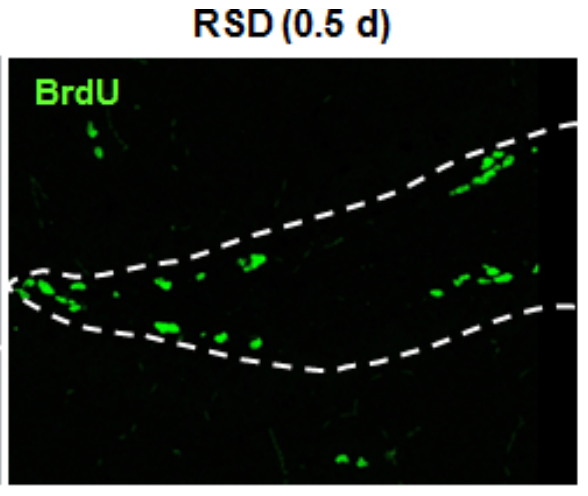

$\operatorname{CON}(0.5 \mathrm{~d})$

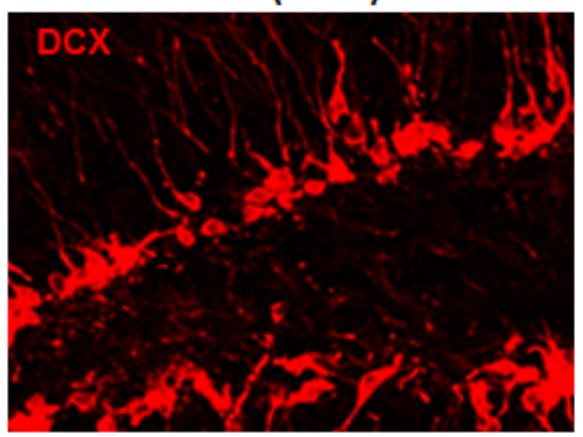

B.

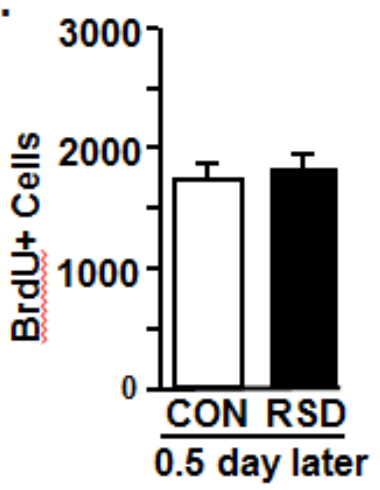

D.

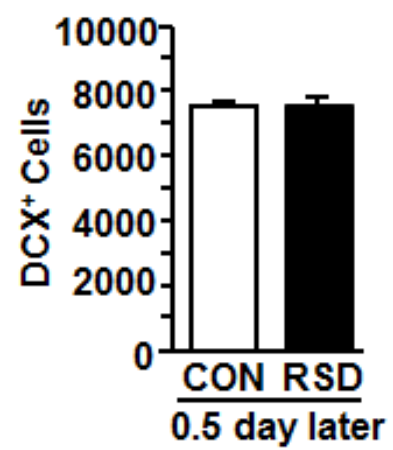

E.

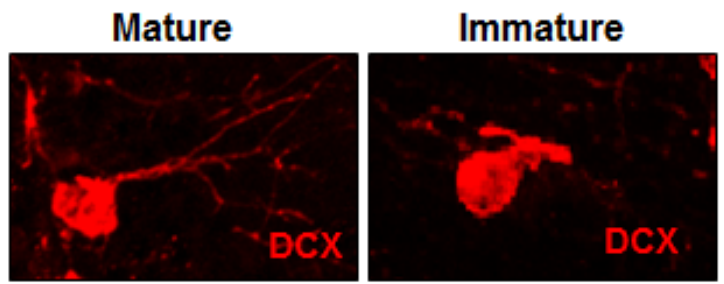

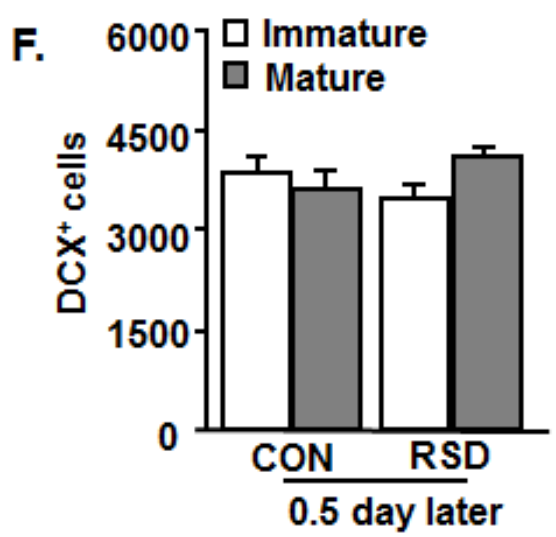

R SD did not affect the number of proliferating cells or young neurons in the dentate gyrus. $M$ ice were subjected to 6 cycles of social defeat or left undisturbed as controls. B rdU was injected during the last three cycles of social defeat, and brains were collected and processed 14 $\mathrm{h}$ later. A) Representative images of B rdU labeling in the dentate gyrus for Con and RSD mice. B) Quantification of BrdU labeling in the dentate gyrus for Con and RSD mice. C) Representative images of DCX in the dentate gyrus for Con and RSD mice. D) Quantification of DCX labeling in the dentate gyrus for Con and RSD mice. E) Representative images of mature and immature DCX-positive neurons F). ) quantification of immature DCX labeling and mature DCX labeling in the dentate gyrus for Con and RSD mice. $B$ ars represent the mean \pm SEM . 


\section{Figure 6}

A.

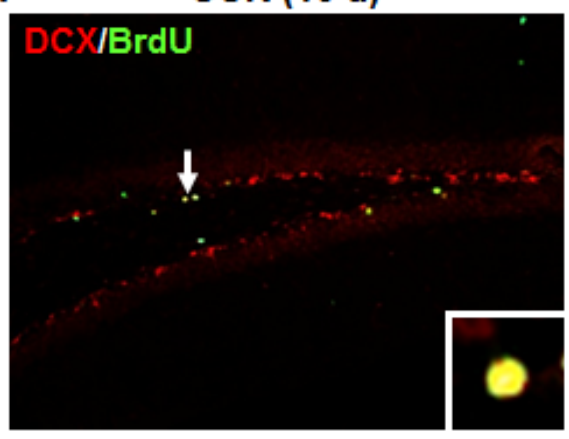

$\operatorname{RSD}(10 \mathrm{~d})$

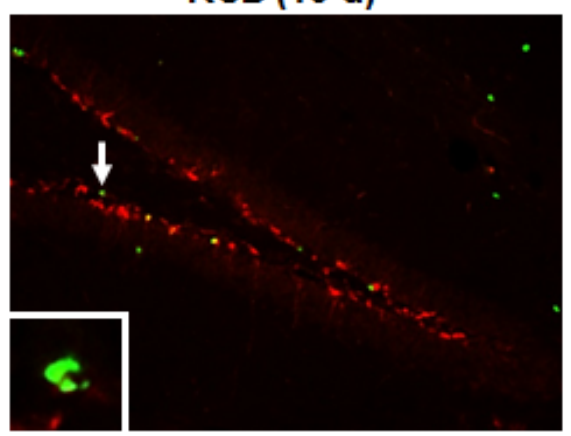

B.

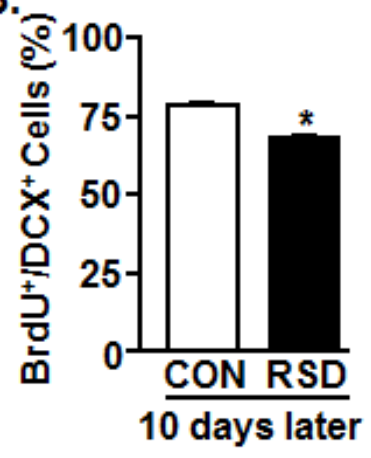

RSD reduced the number of young proliferating neurons in the dentate gyrus. $M$ ice were subjected to 6 cycles of social defeat or left undisturbed as controls. BrdU was injected during the last three cycles, brains were collected and processed $10 \mathrm{~d}$ later. Representative images and quantification of B rdU/DCX double labeling in the dentate gyrus are shown in (A) and (B) respectively. The insets represent a B rdU +/DCX + cell (yellow) and B rdU +/DCX - cell (green). $B$ ars represent the mean \pm SEM. M eans with asterisk $(*)$ are significantly different from CON ( $p$ $<.05)$. 


\section{Figure 7}

A.

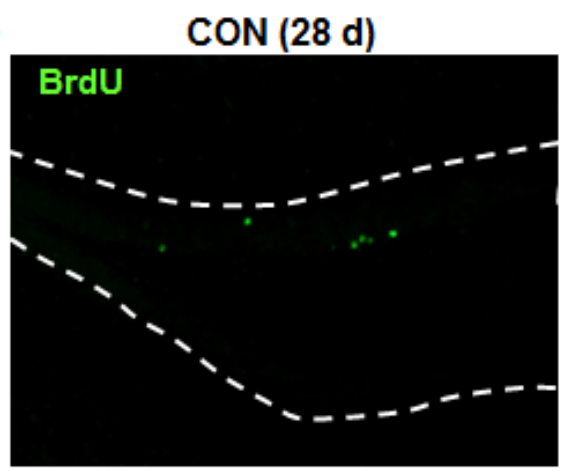

C.

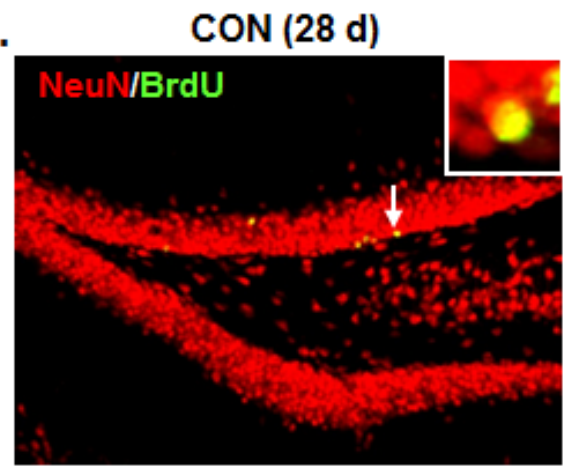

E.

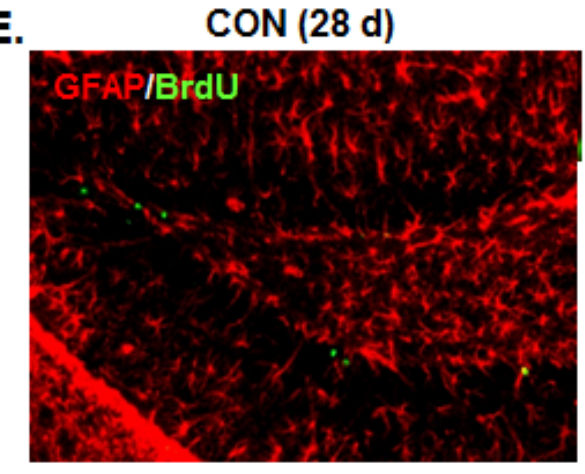

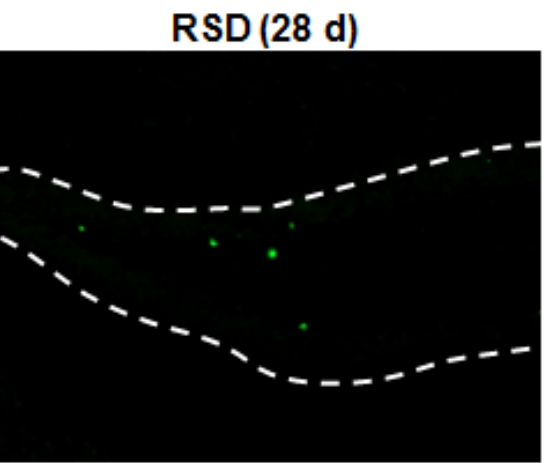

$\operatorname{RSD}(28 \mathrm{~d})$

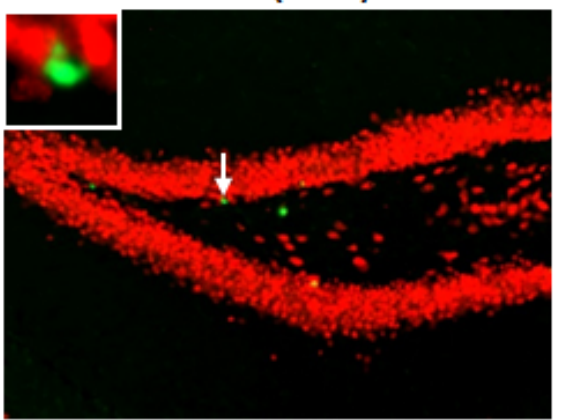

$\operatorname{RSD}(28 \mathrm{~d})$

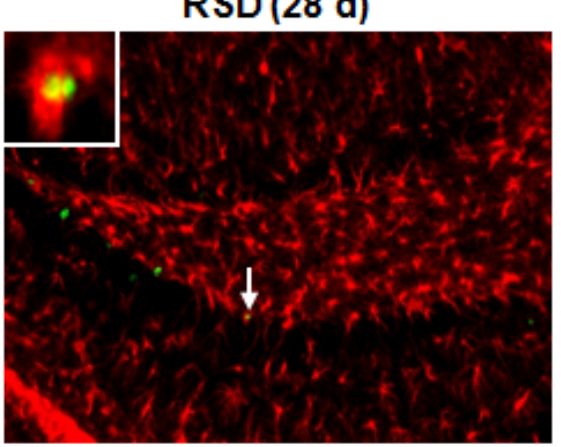

B.

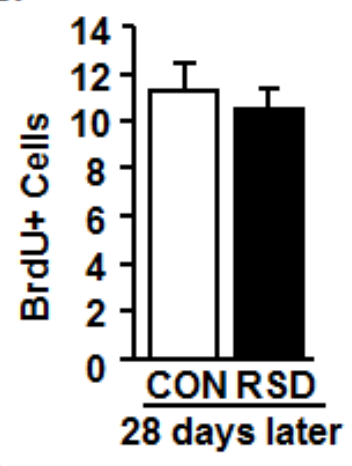

D.

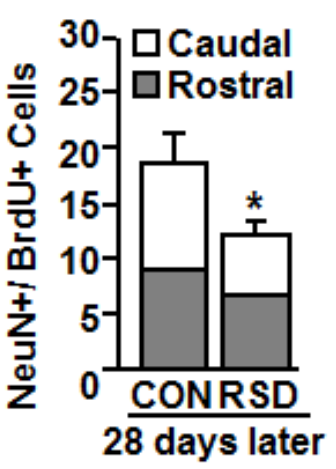

F.

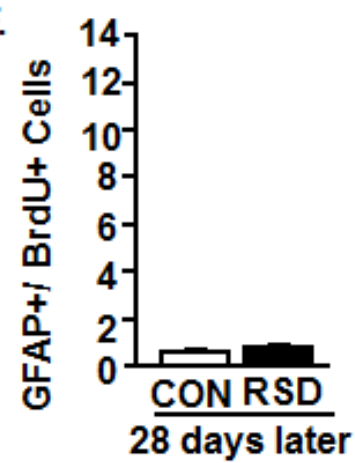

RSD reduced the number of mature neurons but not astrocytes in the dentate gyrus when examined 28 days post-R SD. M ice were exposed to RSD or CON conditions; B rdU was injected during the last three cycles, and mice sacrificed 28 days later. Representative images and quantification of $B$ rdU $(A, B), B$ rdU/N euN $(C, D)$, and $B r d U / G F A P(E, F)$ in the dentate gyrus are shown. $B$ ars represent the mean \pm SEM . M eans with asterisk $(*)$ are significantly different from CON $(p<.05)$. 
Figure 8

A.

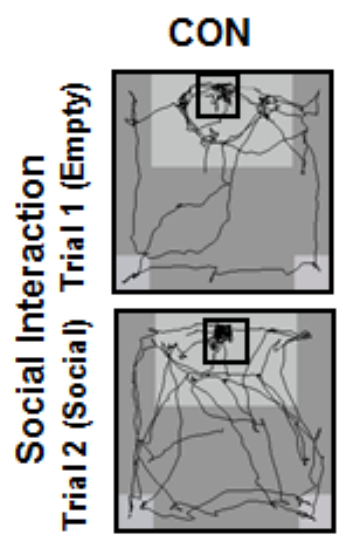

B.



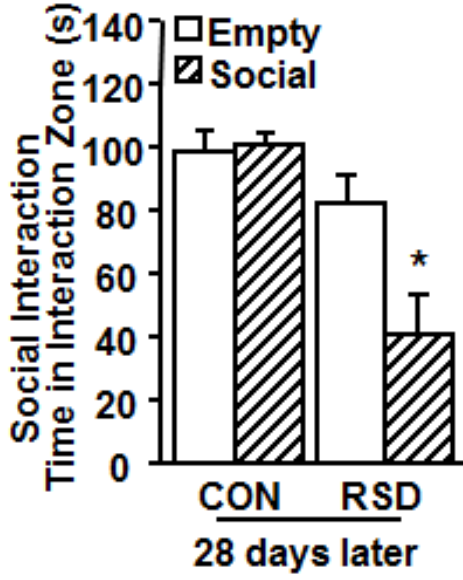

C.

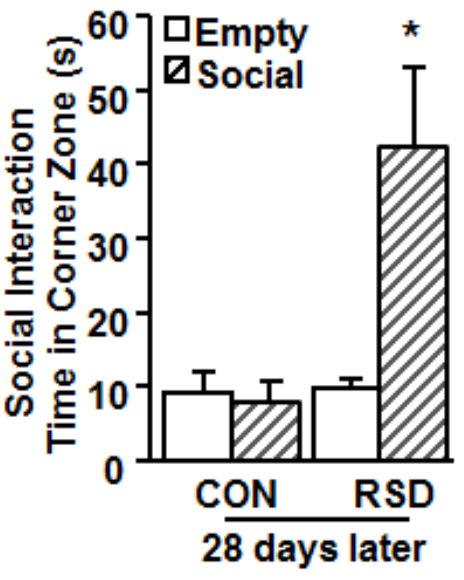

R SD-induced social avoidance behavior was maintained 28 days after stress. M ice exposed to RSD or CON conditions were assessed on the social avoidance test 28 days after RSD. A) Representative paths during empty trial (top) and social trial (bottom) are shown for CON and RSD groups. B) Time spent in the interaction zone and C) Time spent in the corner zone are shown. $B$ ars represent the mean $\pm S E M$. M eans with asterisk $(*)$ are significantly different from $\operatorname{CON}(p<.05)$. 


\section{R eferences}

1. Glaser, R. and J.K. K iecolt-Glaser, Stress-induced immune dysfunction: implications for health. Nat Rev Immunol, 2005. 5(3): p. 243-51.

2. Haroon, E., C.L. Raison, and A.H. M iller, Psychoneuroimmunology meets neuropsychopharmacology: translational implications of the impact of inflammation on behavior. Neuropsychopharmacology, 2012. 37(1): p. 137-62.

3. Vitaliano, P.P., et al., P sychophysiological mediators of caregiver stress and differential cognitive decline. Psychol A ging, 2005. 20(3): p. 402-11.

4. Vitaliano, P.P., et al., D oes caring for a spouse with dementia promote cognitive decline? A hypothesis and proposed mechanisms. J A m Geriatr Soc. 59(5): p. 900-8.

5. W ohleb, E.S., et al., M onocyte trafficking to the brain with stress and inflammation: a novel axis of immune-to-brain communication that influences mood and behavior. Front Neurosci, 2014. 8: p. 447.

6. B rydon, $L$., et al., Psychological stress activates interleukin-1beta gene expression in human mononuclear cells. Brain Behav Immun, 2005. 19(6): p. 540-6.

7. Wohleb, E.S., et al., Stress-induced recruitment of bone marrow-derived monocytes to the brain promotes anxiety-like behavior. J Neurosci, 2013. 33(34): p. 13820-33.


marrow, peripheral blood and spleen. J Neuroimmunol, 2004. 148(1-2): p. 106-15.

9. A vitsur, R., J.L. Stark, and J.F. Sheridan, Social stress induces glucocorticoid resistance in subordinate animals. Horm B ehav, 2001. 39(4): p. 247-57.

10. Powell, N.D., et al., Social stress up-regulates inflammatory gene expression in the leukocyte transcriptome via beta-adrenergic induction of myelopoiesis. Proc $\mathrm{N}$ atl $\mathrm{A}$ cad Sci U S A, 2013. 110(41): p. 16574-16579.

11. W ohleb, E.S., et al., P eripheral innate immune challenge exaggerated microglia activation, increased the number of inflammatory CNS macrophages, and prolonged social withdrawal in socially defeated mice. Psychoneuroendocrinology, 2012. 37(9): p. 1491-505.

12. Wohleb, E.S., et al., ${ }^{2}$-Adrenergic receptor antagonism prevents anxiety-like behavior and microglial reactivity induced by repeated social defeat. J Neurosci, 2011. 31(17): p. 6277-6288.

13. M cEwen, B.S., Stress and hippocampal plasticity. A nnu Rev Neurosci, 1999. 22: p. 10522.

14. K o0, J.W. and R.S. Duman, IL-1beta is an essential mediator of the antineurogenic and anhedonic effects of stress. Proc Natl A cad Sci U S A , 2008. 105(2): p. 751-6.

15. M iller, A.H., V. M aletic, and C.L. Raison, Inflammation and its discontents: the role of cytokines in the pathophysiology of major depression. Biol Psychiatry, 2009. 65(9): p. 732-41.

16. O'Connor, J.C., et al., Lipopolysaccharide-induced depressive-like behavior is mediated by indoleamine 2,3-dioxygenase activation in mice. M ol Psychiatry, 2008.

17. Sawicki, C., et al., Social defeat promotes a reactive endothelium in a brain regiondependent manner with increased expression of key adhesion molecules, selectins and chemokines associated with the recruitment of myeloid cells to the brain. Neuroscience, 2014. 
18. Henry, C.J., et al., M inocycline attenuates lipopolysaccharide (LPS)-induced neuroinflammation, sickness behavior, and anhedonia. J Neuroinflammation, 2008. 5: p. 15.

19. Henry, C.J., et al., Peripheral lipopolysaccharide (LPS) challenge promotes microglial hyperactivity in aged mice that is associated with exaggerated induction of both proinflammatory IL-1beta and anti-inflammatory IL-10 cytokines. Brain Behav Immun, 2009.

20. Quan, N., In-depth conversation: Spectrum and kinetics of neuroimmune afferent pathways. Brain Behav Immun.

21. Cunningham, $C_{\text {., }}$ et al., Systemic Inflammation Induces Acute B ehavioral and Cognitive Changes and Accelerates N eurodegenerative Disease. Biol Psychiatry, 2008.

22. Hinwood, M., et al., Chronic stress induced remodeling of the prefrontal cortex: structural re-organization of microglia and the inhibitory effect of minocycline. Cereb Cortex. 23(8): p. 1784-97.

23. Ekdahl, C.T., Z. K okaia, and O. Lindvall, Brain inflammation and adult neurogenesis: the dual role of microglia. Neuroscience, 2009. 158(3): p. 1021-9.

24. Ekdahl, C.T., et al., Inflammation is detrimental for neurogenesis in adult brain. Proc Natl A cad Sci U S A, 2003. 100(23): p. 13632-7.

25. Green, H.F., et al., A role for interleukin-1beta in determining the lineage fate of embryonic rat hippocampal neural precursor cells. M ol Cell N eurosci. 49(3): p. 311-21.

26. Y irmiya, R. and I. Goshen, I mmune modulation of learning, memory, neural plasticity and neurogenesis. Brain B ehav Immun, 2011. 25(2): p. 181-213.

27. Wohleb, E.S., et al., Stress-induced recruitment of bone marrow-derived monocytes to the brain promotes anxiety-like behavior. J Neurosci, 2013. 33(34): p. 13820-13833.

28. M orris, R., D evelopments of a water-maze procedure for studying spatial learning in the rat. J Neurosci M ethods, 1984. 11(1): p. 47-60.

29. Sparkman, N.L., et al., Interleukin-6 facilitates lipopolysaccharide-induced disruption in working memory and expression of other proinflammatory cytokines in hippocampal neuronal cell layers. J Neurosci, 2006. 26(42): p. 10709-16.

30. Wohleb, E.S., et al., Re-establishment of Anxiety in Stress-Sensitized M ice Is Caused by M onocyte Trafficking from the Spleen to the Brain. Biol Psychiatry, 2014.

31. Paxinos, G. and K. Franklin, The mouse brain in stereotaxic coordinates. 3rd edition. 2008.

32. Hein, A.M., et al., Sustained hippocampal IL-1beta overexpression impairs contextual and spatial memory in transgenic mice. Brain B ehav Immun. 24(2): p. 243-53.

33. Wohleb, E.S., et al., Re-establishment of Anxiety in Stress-Sensitized M ice Is Caused by Monocyte Trafficking from the Spleen to the Brain. Biol Psychiatry.

34. Ben M enachem-Zidon, 0 ., et al., Intrahippocampal transplantation of transgenic neural precursor cells overexpressing interleukin-1 receptor antagonist blocks chronic isolation-induced impairment in memory and neurogenesis. Neuropsychopharmacology, 2008. 33(9): p. 2251-62.

35. Zhao, C., et al., D istinct morphological stages of dentate granule neuron maturation in the adult mouse hippocampus. J Neurosci, 2006. 26(1): p. 3-11.

36. D'M ello, C., T. Le, and M.G. Swain, Cerebral microglia recruit monocytes into the brain in response to tumor necrosis factoralpha signaling during peripheral organ inflammation. J Neurosci, 2009. 29(7): p. 2089-102. 
37. Frank, M.G., et al., M icroglia serve as a neuroimmune substrate for stress-induced potentiation of CNS pro-inflammatory cytokine responses. B rain B ehav Immun, 2007. 21(1): p. 47-59.

38. Lehmann, M.L., et al., G lucocorticoids orchestrate divergent effects on mood through adult neurogenesis. J Neurosci, 2013. 33(7): p. 2961-72.

39. Fanselow, M.S. and H.W. Dong, Are the dorsal and ventral hippocampus functionally distinct structures? Neuron, 2010. 65(1): p. 7-19.

40. Bannerman, D.M., et al., Regional dissociations within the hippocampus--memory and anxiety. Neurosci Biobehav Rev, 2004. 28(3): p. 273-83.

41. Donahue, R.J., et al., E ffects of striatal D eltaF osB overexpression and ketamine on social defeat stress-induced anhedonia in mice. Biol Psychiatry, 2014. 76(7): p. 550-8.

42. V oorhees, J.L., et al., Prolonged restraint stress increases IL-6, reduces IL-10, and causes persistent depressive-like behavior that is reversed by recombinant IL-10. PLoS One, 2013. 8(3): p. e58488.

43. Lagace, D.C., et al., Adult hippocampal neurogenesis is functionally important for stressinduced social avoidance. Proc Natl A cad Sci U S A, 2010. 107(9): p. 4436-41. 